PHYSICAL REVIEW D 89, 033006 (2014)

\title{
Study of the standard model Higgs boson partial widths and branching fractions
}

\author{
Leandro G. Almeida, ${ }^{1}$ Seung J. Lee, ${ }^{2,3}$ Stefan Pokorski, ${ }^{4}$ and James D. Wells ${ }^{5}$ \\ ${ }^{1}$ LPT, Université Paris-Sud and CNRS, 91405 Orsay Cedex, France \\ ${ }^{2}$ Department of Physics, Korea Advanced Institute of Science and Technology, \\ 335 Gwahak-ro, Yuseong-gu, Daejeon 305-701, Korea \\ ${ }^{3}$ School of Physics, Korea Institute for Advanced Study, Seoul 130-722, Korea \\ ${ }^{4}$ Faculty of Physics, Institute of Theoretical Physics, University of Warsaw, Hoża 69, Warsaw, Poland \\ ${ }^{5}$ CERN Theoretical Physics, CH-1211 Geneva 23, Switzerland, and Physics Department,
}

University of Michigan, Ann Arbor, Michigan 48109, USA

(Received 9 December 2013; published 12 February 2014)

\begin{abstract}
The discovery of the Higgs boson, with a mass known to be better than the percent level, enables precision Higgs boson analyses for the first time. Toward this goal, we define an expansion formalism of the Higgs boson partial widths and branching fractions that facilitates such studies. This expansion yields the observables as a perturbative expansion around reference values of Standard Model input observables (quark masses, QCD coupling constant, etc.). We compute the coefficients of the expansion using state-ofthe-art results. We also study the various sources of uncertainties in computing the partial widths and branching fractions more precisely. We discuss the impact of these results with efforts to discern new physics through precision Higgs boson studies.
\end{abstract}

DOI: $10.1103 /$ PhysRevD.89.033006

PACS numbers: 14.80.Bn, 14.65.Ha, 12.60.-i

\section{INTRODUCTION}

With the discovery of the Higgs boson [1], particle physics is entering a new era of precision studies of the Higgs sector. The observables are many and include the Higgs boson mass, its total decay width, its spin, its decay branching fractions to Standard Model (SM) particles, its possible decay branching fractions to other exotic final states, and its various production rates at colliders. All of these observables will be studied carefully in time.

The theory under primary consideration in this article is the Standard Model. The subpercent-level determination of the Higgs boson mass now enables a complete set of input observables whereby any perturbative high-energy observable involving the Higgs boson can be predicted.

In this article, our focus is on the careful exposition of the decay partial widths and branching fractions of a SM Higgs boson with mass near $126 \mathrm{GeV}$. Our goal is to provide state-of-the-art formulas that can be used in any precision electroweak analysis to investigate compatibility of the data with the SM predictions in these most fundamental and sensitive observables. Other calculations exist in the literature, ${ }^{1}$ most notably from the computer program HDECAY [2]; however, we wish to provide an independent calculation that includes the latest advances and allows us to vary the renormalization scale in all parts of the computations. This flexibility will be useful in later discussions regarding uncertainties. We also aim to detail the errors that each input into the computation propagates

\footnotetext{
${ }^{1}$ For a basic review, see [4] and references therein.
}

to the final answer for each observable [3]. In some cases, these uncertainties are large, and constitute a limitation to how sensitive experimental measurements can be in determining the underlying theory parameters. Finally, we discuss some implications for physics beyond the SM sensitivities in precision Higgs studies.

\section{INPUT OBSERVABLES}

There are an infinite number of SM observables that can be defined, yet any one of them, in principle, can be computed precisely once a fixed, complete, independent, and finite set of input observables is specified. A convenient set of input observables is

$$
\left\{m_{H}, M_{Z}, \Delta \alpha_{\text {had }}^{(5)}, \alpha_{S}\left(M_{Z}\right), m_{f}\right\},
$$

where $m_{f}$ represents the list of fermion masses of the SM: $m_{t}, m_{b}, m_{c}, m_{\tau}, m_{\mu}$, etc. We are ignoring flavor angles for the purposes of the present discussion. We can specify $\alpha\left(M_{Z}\right)$ by $\Delta \alpha_{\text {had }}^{(5)}$ alone. The relation between the two is

$$
\alpha\left(M_{Z}\right)=\frac{\alpha}{1-\Delta \alpha_{e, \mu, \tau}-\Delta \alpha_{t}-\Delta \alpha_{\mathrm{had}}^{(5)}},
$$

where $\alpha$ is the well-known $1 / 137.036$ and $\Delta \alpha_{e, \mu, \tau}$ and $\Delta \alpha_{t}$ are perturbatively calculable and known very accurately [5]. The weak link to a more precise knowledge of $\alpha\left(M_{Z}\right)$ is $\Delta \alpha_{\text {had }}^{(5)}$, which is extracted mostly via dispersion relations from $e^{+} e^{-} \rightarrow$ hadrons data at low energy. Since all the 
uncertainty of $\alpha\left(M_{Z}\right)$ originates in $\Delta \alpha_{\text {had }}^{(5)}$, it is customary to specify that value as the input, which by Eq. (2) then dictates the value of $\alpha\left(M_{Z}\right)$. The values of all the input parameters are given in Table I.

The $S U(2) \times U(1) \rightarrow U(1)_{E M}$ gauge structure and symmetry breaking make the prediction of $M_{W}$ from other input observables an important test of the theory. Nevertheless, for us, $M_{W}$ shows up in the partial width calculations as a kinematic mass in the propagator of a loop expression $(H \rightarrow b \bar{b}$ loops, etc.) or as the final state mass in a phase space computation $\left(H \rightarrow W W^{*}\right)$. Since it very directly appears in these computations, one might be tempted to choose it as an input parameter to the calculations of precision Higgs observables. This is legitimate and acceptable in principle. One can exchange, for example, $m_{t}$ for $M_{W}$ as an input. However, several complications arise. The choice of $M_{W}$ as an input may simplify the computation in some ways but makes it more complicated in other ways (e.g., in utilizing self-consistent top mass). More importantly, there is a risk that by doing so, one can choose incompatible sets of input parameters for predictions of different sets of observables. For example, in computing precision Z-decay and LEP2 observables, one might choose the standard set of inputs that does not include $M_{W}$, whereas for Higgs sector observables, one might choose a set of inputs that includes $M_{W}$. Making a comparison between $B(Z \rightarrow b \bar{b})$ and $B(H \rightarrow b \bar{b})$, for example, when testing the SM becomes impossible unless an equivalence dictionary between the two sets is clearly specified and self-consistent, equivalent sets of inputs are chosen. For this reason, we specify one set of input observables for all computations, and that set is the one where $M_{W}$ is an output.

Now that we have established our convention that $M_{W}$ is an output observable, when the $W$ mass appears in formulas below, we should view it as a short-hand notation for the full computation of the $W$ mass within the theory in terms of our agreed-upon inputs. In the SM this substitution is

$$
\begin{aligned}
M_{W} \stackrel{\mathrm{SM}}{\rightarrow} & (80.368 \mathrm{GeV})\left(1+1.42 \delta M_{Z}+0.21 \delta G_{F}-0.43 \delta \alpha\right. \\
& \left.+0.013 \delta M_{t}-0.0011 \delta \alpha_{S}-0.00075 \delta M_{H}\right) .
\end{aligned}
$$

This formula is obtained by expanding results in [8], which we have independently checked. Numerical evaluation was done using the reference values of the input parameters given in Table I. The definition of $\delta \tau$ is $\delta \tau \equiv\left(\tau-\tau_{\text {ref }}\right) / \tau_{\text {ref }}$.

One ultimate goal of this work is to survey state-of-the art calculations in order to test the SM. The proper way to test any theory is to compute all the observables and subject them to a global $\chi^{2}$ likelihood test, where

$$
\chi^{2}=\sum_{i}\left(\frac{\mathcal{O}^{\text {th }}-\mathcal{O}^{\text {expt }}}{\Delta \mathcal{O}_{i}^{\text {expt }}}\right)^{2} .
$$

One should take all the correlations amongst observables into account as well [9]. Upon computing the $\chi^{2}$ it is then possible to ask statistical inference questions about the value. For example, is the $\chi^{2}$ per degree of freedom indicating that the theory is compatible with the data at some confidence level? In such a procedure, it makes no difference what independent input parameters one uses: there is an infinite set of possibilities that are equally good and the answer to any well-defined question is the same regarding confidence in a theory or range of values predicted for an observable given the data, etc. The results that we present will also enable a very quick determination of our present abilities to determine the measurement couplings of SM particles to the Higgs boson. Each of the input observables has a number of uncertainties associated with it, and when these errors propagate, there will be uncertainties for the predictions of the partial widths and branching fraction observables. At the moment, the predicted uncertainties (a few percent or less) are much smaller than the current measured uncertainties (tens of percent), but in the future this limiting theory precision will become important as experiments improve. We note that in the current experimental situation the prediction uncertainties would be nearly the same had we chosen $M_{W}$ rather than $m_{t}$ as an input. Indeed it is somewhat accidental that the target observables have nearly the same small prediction uncertainty for either choice.

In the following sections, we will present the computations for each of the important decay-mode partial widths of the SM Higgs boson. The results are presented here in

TABLE I. Reference values for the input observables [see Eq. (1)] chosen for computation of the widths and branching ratios of the Higgs boson. Units are in GeVs for the masses. All the reference values except for $m_{H}$ [6] and $\alpha\left(M_{Z}\right)$ (or $\left.\Delta \alpha_{\text {had }}^{(5)}\right)$ are given by [7]. $\alpha_{S}\left(M_{Z}\right)$ is taken to be the world average value. As explained in the text, specifying $\alpha\left(M_{Z}\right)$ and $\Delta \alpha_{\text {had }}^{(5)}$ (from the Winter 2012 plots of the LEP Electroweak Working Group [5]) in this table is redundant but it is done for the convenience of the reader.

\begin{tabular}{lccc}
\hline \hline$m_{H}$ & $125.7(4)$ & pole mass $m_{t}$ & $173.5(10)$ \\
pole mass $m_{c}$ & $1.67(7)$ & pole mass $m_{b}$ & $4.78(6)$ \\
pole mass $M_{Z}$ & $91.1535(21)$ & $G_{F}$ & $1.1663787(6) \times 10^{-5}$ \\
pole mass $m_{\tau}$ & $1.77682(16)$ & $\alpha_{S}\left(M_{Z}\right)$ & $0.1184(7)$ \\
$\alpha\left(M_{Z}\right)$ & $1 / 128.96(2)$ & $\Delta \alpha_{\text {had }}$ & $0.0275(1)$ \\
\hline \hline
\end{tabular}


order to show the origin of our subsequently derived expansions of these partial widths and branching fractions in terms of small deviations away from measured reference values of the input observables.

\section{HIGGS BOSON PARTIAL WIDTHS}

In this section, we describe the procedures by which we compute the partial widths of the Higgs boson decays. In each case they are taken from state-of-the-art computations within the literature. It is intended that the reader can reproduce all of our results by following the instructions we give below.

\section{A. Higgs boson decays to $W W^{*}$ and $Z Z^{*}$}

The interaction between the Higgs and the electroweak vector bosons can be best probed through its direct decay into vector bosons. The mass of the Higgs boson at 126 $\mathrm{GeV}$ excludes the decay into two on-shell electroweak vector bosons, leaving the following decays: $H \rightarrow V^{(*)} V^{*}$. The width for a Higgs boson decaying into $V^{*} V$ is given at lowest order in [10].

The vector bosons further decay into fermions, and there are interferences between the intermediate off-shell vector bosons as a consequence. This result is known at $\mathcal{O}\left(\alpha_{S}\right)$ and at $\mathcal{O}(\alpha)$ and its calculation is described in [11]. The results were implemented in a Monte Carlo generator, Prophecy $4 f$ [12], which is what we use to compute the $W W^{*}$ and $Z Z^{*}$ partial width values.

\section{B. Higgs boson decays to $\gamma \gamma$ and $Z \gamma$}

The higher-order contributions to $\Gamma(H \rightarrow \gamma \gamma)$ are known to Next-to-next-to-leading order (NNLO) $\mathcal{O}\left(\alpha_{S}^{2}\right)$ in QCD [13], and at Next-to-leading order (NLO) in purely electroweak (EW) corrections [14]. We parametrize the results as

$$
\begin{aligned}
\Gamma(H \rightarrow \gamma \gamma)= & \Gamma_{\mathrm{LO}}^{\gamma \gamma}+\frac{\alpha}{\pi} \Gamma_{\mathrm{NLO}-\mathrm{EW}}^{\gamma \gamma}+\left(\frac{\alpha_{S}}{\pi}\right) \Gamma_{\mathrm{NLO}-\mathrm{QCD}}^{\gamma \gamma} \\
& +\left(\frac{\alpha_{S}}{\pi}\right)^{2} \Gamma_{\mathrm{NNLO}-\mathrm{QCD}}^{\gamma \gamma},
\end{aligned}
$$

where $\alpha=\alpha_{\mathrm{QED}}\left(m_{H}^{2}\right)$ at one loop, and $\alpha_{S}=\alpha_{S}\left(m_{H}^{2}\right)$ at three loops are as provided by RunDec [15]. The results for $\Gamma_{\text {NNLO-QCD }}$ are obtained from [13], and for consistency, we also use its results for $\Gamma_{\mathrm{NLO}-\mathrm{QCD}}$. For both orders we use the expansion in $x_{t}=m_{H}^{2} /\left(4 m_{t}^{2}\right)$ to $\mathcal{O}\left(x_{t}^{5}\right)$. For $\Gamma_{\mathrm{NLO}-\mathrm{EW}}$ we interpolate the results of [14] to the same order.

For the prediction of $\Gamma(H \rightarrow Z \gamma)$ we use the results of [16], which give the contributions to lowest order with an additional contribution from QCD involving top quark loops. The result is parametrized below:

$$
\Gamma(H \rightarrow Z \gamma)=\Gamma_{\mathrm{LO}}^{Z \gamma}+\frac{\alpha_{S}}{\pi} \Gamma_{\text {aprox-NLO}}^{Z \gamma} .
$$

Here, $\Gamma_{\text {aprox-NLO }}^{Z_{\gamma}}$ is the additional contribution from QCD to the top quark loop. This is achieved by shifting the top amplitude in the lowest order contribution [16].

\section{Higgs boson decays to gluons}

Similarly, the partial width of the Higgs boson decaying into gluons is given at NNLO, $\mathcal{O}\left(\alpha_{S}^{2}\right)$ in the full SM theory [17]. While the result is also known at NNNLO in the effective theory $[18,19]$, resulting from integrating out the top quark, we use only the results from the full SM computation.

For the electroweak corrections, we make use of the numerical results of [20] and extrapolate them to $\mathcal{O}\left(x_{t}^{4}\right)$, where $x_{t}=m_{H}^{2} /\left(4 m_{t}^{2}\right)$. We use the three-loop result for $\alpha_{S}$, running to the proper scale choice. The scales are chosen to be $m_{H}$, with the exception of the electroweak corrections, whose scale dependences are not provided, but were indicated to be small in [20].

\section{Higgs boson decays to quarks}

The dominant decay for the Higgs boson is directly into $b \bar{b}$. For its partial width we use the results of [21], which provide the nonpower-suppressed corrections to $\mathcal{O}\left(\alpha_{S}^{4}\right)$. We obtain $\mathcal{O}\left(G_{F} m_{t}^{4}\right)$ corrections from [22]. Higher-order logarithmic corrections are absorbed into the running quark masses. All masses are evolved using functions obtained from RunDec [15] to the appropriate loop order. In the case of the $H \rightarrow b \bar{b}$ partial width, we need to evolve the $\overline{\mathrm{MS}}$ mass, $m_{b}$, to three loops, given the accuracy of the calculation. In the case of $c \bar{c}$, we make use of the electroweak corrections found in [22], while keeping the same order in QCD as $b \bar{b}$. The scale dependence to order $\mathcal{O}\left(\alpha_{S}^{3}\right)$ is given in [23,24] for the diagonal correlators. We make use of the result at $\mathcal{O}\left(\alpha_{S}^{4}\right)$ at $s=m_{h}^{2}$, and $n_{f}=5$ from [21], and, with the renormalization group equations, extend the scale dependence to $\mathcal{O}\left(\alpha_{S}^{4}\right)$. The one-loop pure electroweak contributions were obtained by $[25,26]$. We use the full analytical result for its dependence on all on-shell quark masses (with $\left.m_{u, d, s}=0\right)$ and lepton masses $\left(m_{e}=0\right)$. The $W$ mass is determined as described above, and we subtract the leading contribution in $G_{F} m_{t}^{2}$ to avoid double counting from the contributions mentioned above.

\section{E. Higgs boson to leptons}

For the partial decay width into two leptons we make use of the next-to-leading-order QCD corrections up to $\mathcal{O}\left(\alpha_{s}^{2} G_{F} m_{t}^{2}\right)$ and two-loop electroweak corrections in $[22,27]$.

\section{EXPANSION OF PARTIAL WIDTHS AND UNCERTAINTIES}

Now that we have the full expressions for the partial widths of Higgs boson decays we are in the position to Taylor expand these equations around the input 
TABLE II. Running M $\bar{S}$ masses for the heavy quarks at three loops at the scale $\mu=m_{H}, m_{H} / 2$ and $2 m_{H}$ from the program RunDec [15], which is used for the Higgs boson decaying into quarks. Pole-mass inputs are taken from Table I. The parametric uncertainty on the running mass at $\mu=m_{H}$ from $1 \sigma$ uncertainty $\left(\sigma_{m}\right)$ in the pole mass is defined to be $P_{m}(\Delta m)=\left\{m_{+}\left(m_{H}\right)+\right.$ $\left.m_{-}\left(m_{H}\right)\right\} /\left\{2 m\left(m_{H}\right)\right\}$, where $m_{ \pm}\left(m_{H}\right)$ is computed using $m_{\text {pole }}=m_{\text {ref }} \pm \sigma_{m}$. The scale dependence of the running mass is canceled in higher-order loop calculations, as can be seen later for scale-dependence uncertainties.

\begin{tabular}{lcc}
\hline \hline Quark & at $\mu=m_{H}\left(m_{H} / 2,2 m_{H}\right)$ & $P_{m}(\Delta m)$ \\
\hline$m_{c}(\mu)$ & $0.576(0.612,0.546) \mathrm{GeV}$ & $7.53 \%$ \\
$m_{b}(\mu)$ & $2.68(2.84,2.54) \mathrm{GeV}$ & $1.62 \%$ \\
$m_{t}(\mu)$ & $167(177,158) \mathrm{GeV}$ & $0.63 \%$ \\
\hline \hline
\end{tabular}

observables. This expansion is made possible by the fact that with the discovery of the Higgs boson, and knowledge of its mass, all input observables are now known to good enough accuracy to render an expansion of this nature useful and accurate.

We represent the partial width expansion by

$$
\Gamma_{H \rightarrow X}=\Gamma_{X}^{(\mathrm{ref})}\left(1+\sum_{i} a_{\tau_{i}, X} \overline{\delta \tau_{i}}\right),
$$

where

$$
\bar{\delta}_{i}=\frac{\tau_{i}-\tau_{i, \mathrm{ref}}}{\tau_{i, \mathrm{ref}}}
$$

and $\tau_{i}$ are the input observables [Eq. (1)] for the calculation. The total width is the sum of all the partial widths; for convenience we present dedicated expansion parameters for that as well:

$$
\Gamma_{\mathrm{tot}}=\sum_{X} \Gamma_{H \rightarrow X}=\Gamma_{\mathrm{tot}}^{(\mathrm{ref})}\left(1+\sum_{i} a_{\tau_{i}, \mathrm{tot}} \overline{\delta \bar{\tau}_{i}}\right) .
$$

For many of our parameters and observables we would like to know the relative uncertainty due to variations in the input parameters or variations of scale. The "percent relative uncertainty," $P_{Q}$, of a parameter or observable $Q$ from its central reference value $Q_{0}$ is defined as

$$
Q=Q_{0}\left(1+0.01 P_{Q}\right)
$$

TABLE IV. The estimates for percent relative uncertainty on the partial widths from parametric and scale-dependence uncertainties. Parametric uncertainties arise from incomplete knowledge of the input observables for the calculation (i.e., errors on $m_{c}, \alpha_{s}$, etc.). For parametric uncertainties, we put an additional number in parentheses, which is the value it would have if the Higgs boson mass uncertainty were $0.1 \mathrm{GeV}$ (instead of $0.4 \mathrm{GeV}$ ). Scale-dependence uncertainties are indicative of not knowing the higher-order terms in a perturbative expansion of the observable. These uncertainties are estimated by varying $\mu$ from $m_{H} / 2$ to $2 m_{H}$. More details on the precise meaning of the entries of this table are found in the text of Sec. IV. Errors below $0.01 \%$ are represented in this table as 0 .

\begin{tabular}{lccc}
\hline \hline & $P_{\Gamma}^{ \pm}$(par.add. $)$ & $P_{\Gamma}^{ \pm}$(par.quad. $)$ & $\left(P_{\Gamma}^{+}, P_{\Gamma}^{-}\right)(\mu)$ \\
\hline Total & $4.41(3.33)$ & $2.43(2.00)$ & $(0.06,0.09)$ \\
$g g$ & $2.57(1.88)$ & $1.74(1.50)$ & $(0.01,0.04)$ \\
$\gamma \gamma$ & $1.45(0.42)$ & $1.38(0.35)$ & $(1.31,0.60)$ \\
$b \bar{b}$ & $4.94(4.75)$ & $3.54(3.53)$ & $(0.31,0.02)$ \\
$c \bar{c}$ & $20.75(20.56)$ & $15.99(15.99)$ & $(0.43,0.32)$ \\
$\tau^{+} \tau^{-}$ & $0.36(0.13)$ & $0.32(0.09)$ & $(0.01,0.01)$ \\
$W W^{*}$ & $4.41(1.14)$ & $4.97(1.25)$ & $(0.25,0.31)$ \\
$Z Z^{*}$ & $4.90(1.25)$ & $4.42(1.11)$ & $(0 ., 0)$. \\
$Z \gamma$ & $3.56(0.92)$ & $3.52(0.88)$ & $(0.56,0.23)$ \\
$\mu^{+} \mu^{-}$ & $0.34(0.11)$ & $0.32(0.08)$ & $(0.03,0.03)$ \\
\hline \hline
\end{tabular}

If the errors are asymmetric then $P_{Q}^{+}$designates the positive percent relative error that increases the absolute value of $Q$, and $P_{Q}^{-}$designates the negative percent relative error that decreases the absolute value of $Q$. If the positive and negative errors are symmetric then we can combine and label it as $P_{Q}^{ \pm}$.

In the computation we need to know the quark masses at the scale of the Higgs boson mass. The bottom and charm quark masses are evolved to $\mu=m_{H}$ using renormalization group techniques. For consistency, and to avoid confusion, we use the program RunDec [15], which provides the MS evolution up to $\mathcal{O}\left(\alpha_{S}^{4}\right)$. The values at $\mu=m_{H}$ are provided in Table II for easy comparisons.

The partial width expansions are given in Table III, where the reference values $\Gamma_{0}^{(\text {ref })}$ and expansion coefficients of Eq. (7) are computed using the central values of the input observables of Table I. Table IV gives the estimated

\begin{tabular}{|c|c|c|c|c|c|c|c|c|c|c|}
\hline & $\Gamma_{X}^{(\operatorname{Ref})} / \mathrm{GeV}$ & $a_{m_{t}, X}$ & $a_{m_{H}, X}$ & $a_{\alpha\left(M_{Z}\right), X}$ & $a_{\alpha_{S}\left(M_{Z}\right), X}$ & $a_{m_{b}, X}$ & $a_{M_{Z}, X}$ & $a_{m_{c}, X}$ & $a_{m_{\tau}, X}$ & $a_{G_{F}, X}$ \\
\hline Total & $3.96 \times 10^{-3}$ & $-3.48 \times 10^{-2}$ & 4.53 & $4.53 \times 10^{-1}$ & -1.35 & & -3.49 & $9.05 \times 10^{-2}$ & $1.3 \times 10^{-1}$ & $8.43 \times 10^{-1}$ \\
\hline$g g$ & $3.57 \times 10^{-4}$ & $-1.62 \times 10^{-1}$ & 2.89 & 0 & 2.49 & $-7.1 \times 10^{-2}$ & $3.77 \times 10^{-1}$ & 0 & 0 & 1 \\
\hline$\gamma \gamma$ & $1.08 \times 10^{-5}$ & $-2.73 \times 10^{-2}$ & 4.32 & 2.28 & $1.8 \times 10^{-2}$ & $9.01 \times 10^{-3}$ & -1.85 & 0 & 0 & $7.24 \times 10^{-1}$ \\
\hline$b \bar{b}$ & $2.17 \times 10^{-3}$ & $8.11 \times 10^{-3}$ & $8.09 \times 10^{-1}$ & $2.94 \times 10^{-2}$ & -2.46 & 2.57 & $-4.75 \times 10^{-1}$ & 0 & 0 & $9.53 \times 10^{-1}$ \\
\hline$c \bar{c}$ & $9.99 \times 10^{-5}$ & $-4.55 \times 10^{-2}$ & $7.99 \times 10^{-1}$ & $7 \times 10^{-3}$ & -9.17 & 0 & -1.141 & 3.59 & 0 & $9.7 \times 10^{-1}$ \\
\hline$\tau^{+} \tau^{-}$ & $2.58 \times 10^{-4}$ & $2.74 \times 10^{-2}$ & $9.95 \times 10^{-1}$ & $-2.37 \times 10^{-2}$ & $-2.15 \times 10^{-3}$ & 0 & $-1.61 \times 10^{-2}$ & 0 & 2.01 & 1.02 \\
\hline$W W^{*}$ & $9.43 \times 10^{-4}$ & $-1.13 \times 10^{-1}$ & $1.37 \times 10^{1}$ & 1.82 & $9.04 \times 10^{-3}$ & 0 & $-1.21 \times 10^{1}$ & 0 & 0 & $2.49 \times 10^{-1}$ \\
\hline$Z Z^{*}$ & $1.17 \times 10^{-4}$ & $2.28 \times 10^{-2}$ & $1.53 \times 10^{1}$ & $-3.67 \times 10^{-1}$ & $-1.82 \times 10^{-3}$ & 0 & $-1.12 \times 10^{1}$ & 0 & 0 & 2.53 \\
\hline$Z \gamma$ & $6.88 \times 10^{-6}$ & $-1.54 \times 10^{-2}$ & $1.11 \times 10^{1}$ & $3.81 \times 10^{-1}$ & 0 & $-9.76 \times 10^{-3}$ & -4.82 & 0 & 0 & 2.62 \\
\hline$\mu^{+} \mu^{-}$ & $8.93 \times 10^{-7}$ & $4.84 \times 10^{-2}$ & $9.92 \times 10^{-1}$ & $-4.59 \times 10^{-2}$ & $-2.2 \times 10^{-3}$ & 0 & $-1.62 \times 10^{-2}$ & 0 & 0 & 1.02 \\
\hline
\end{tabular}
parametric and scale-dependence uncertainties on the

TABLE III. Reference values for the partial widths at the central values of the parameters given in Table I along with values for $a_{\tau_{i}, X}$ as defined by Eq. (7). $V V^{*}$ partial decay widths are calculated by Prophecy $4 f$. 
partial width values of Table III. The uncertainties are expressed as percent relative uncertainties according to the definition in Eq. (10). The meaning of $P_{\Gamma}^{ \pm}$(par.add.) is that all input parameters have been allowed to range over their $1 \sigma$ errors and the maximum percent relative errors are recorded. The meaning of $P_{\Gamma}^{ \pm}$(par.quad.) is that the uncertainties of each parameter are added in Gaussian quadrature. In other words, $P_{\Gamma_{i}}^{ \pm}$(par.quad. $)=100 \Delta \Gamma_{i} / \Gamma_{i}$, where

$$
\left(\Delta \Gamma_{i}\right)^{2}=\left(\frac{\partial \Gamma_{i}}{\partial m_{t}}\right)^{2}\left(\Delta m_{t}\right)^{2}+\left(\frac{\partial \Gamma_{i}}{\partial \alpha_{s}}\right)^{2}\left(\Delta \alpha_{s}\right)^{2}+\cdots .
$$

The uncertainties in varying the scale parameter $\mu$ in the calculation attempt to capture the uncertainty regarding higher-order corrections. A full calculation at all orders would give a result that does not depend on $\mu$, but a finiteorder calculation does, and the uncertainty of dropping the higher-order calculations is assumed to be approximated reasonably well by noting how much the result changes by varying $\mu$ by a factor of 2 upward and downward: $m_{H} / 2<\mu<2 m_{H}$. The meaning of $P_{\Gamma}^{ \pm}(\mu)$ in Table IV concerns the relative percent uncertainties associated with this scale-dependence algorithm.

\section{EXPANSION OF BRANCHING FRACTIONS AND UNCERTAINTIES}

In the previous section we derived the expansion of the partial widths in terms of small deviations of the input observables from their reference values, and we determined the uncertainties of the partial widths due to input observable uncertainties (parameter uncertainties) and scaledependence uncertainties. The same type of expansion can be done for branching fractions and ratios of branching fractions. To begin with, the expansion for the branching ratios is

$$
\mathrm{B}(H \rightarrow \mathrm{X})=\mathrm{B}(X)^{(\mathrm{ref})}\left(1+\sum_{i} b_{\tau_{i}, X} \overline{\tau_{i}}\right),
$$

where $\tau_{i}$ represents the same parameters as Eq. (1). Expansion parameters $b_{\tau_{i}, X}$ are related to $a_{\tau_{i}, X}$ by

$$
b_{\tau_{i}, X}=a_{\tau_{i}, X}-a_{\tau_{i}, \text { tot }} .
$$

Using the reference parameters from Table I, we display the results of the reference branching ratios and their expansion coefficients in Table V.

The table of expansion coefficients enables us to compute the uncertainty in a final state branching ratio due to each input parameter. The percent uncertainty $\Delta_{i}^{X}$ on branching fraction $B(X)$ due to input parameter $\tau_{i}$ is

$$
\Delta_{i}^{X}=(100 \%) \times\left|b_{\tau_{i}, X}\right| \frac{\Delta \tau_{i}}{\tau_{i}^{\text {ref }}},
$$

where $\Delta \tau_{i}$ are the current experimental uncertainties in input parameter $\tau_{i}$. For example, the percentage uncertainty in the $H \rightarrow g g$ branching fraction is

$$
\Delta_{b}^{g g}=(100 \%)(1.467) \frac{0.06 \mathrm{GeV}}{4.78 \mathrm{GeV}}=1.84 \% .
$$

Each of these calculations have been done and are presented in Table VI. We see most clearly in this table that the uncertainty in the $b$-quark mass input observable constitutes the largest uncertainty in the branching ratio computations. The large uncertainty of the charm quark mass is the decisive contributor to $H \rightarrow c \bar{c}$ uncertainty as well.

\section{A. Ratios of branching ratios}

Experimental observables at colliders are a combination of cross-section times branching fraction, $\sigma B$. Although this combination $\sigma B$ can often be measured to very high accuracy, the extraction of the branching fractions is fraught with experimental and computational complexity in several ways. First, the parton distribution functions are not known with high enough precision to perform a calculation that would match the precision with which the observable ultimately will be measured. Second, there are additional theory uncertainties in the cross section and the definition of the overall observable that make a clean comparison between theory and experiment difficult.

\begin{tabular}{|c|c|c|c|c|c|c|c|c|c|c|}
\hline & $\mathrm{B}(X)^{(\operatorname{Ref})}$ & $b_{m_{t}}$ & $b_{m_{H}}$ & $b_{\alpha\left(M_{Z}\right)}$ & $b_{\alpha_{S}\left(M_{Z}\right)}$ & $b_{m_{b}}$ & $b_{M_{Z}}$ & $b_{m_{c}}$ & $b_{m_{\tau}}$ & $b_{G_{F}}$ \\
\hline$g g$ & $9.03 \times 10^{-2}$ & $-1.27 \times 10^{-1}$ & -1.64 & $-4.45 \times 10^{-1}$ & 3.84 & -1.47 & 3.87 & $-9.05 \times 10^{-2}$ & $-1.30 \times 10^{-1}$ & $1.57 \times 10^{-1}$ \\
\hline$\gamma \gamma$ & $73 \times 10^{-3}$ & $7.46 \times 10^{-3}$ & $-2.1 \times 10^{-1}$ & 1.84 & 1.37 & -1.39 & 1.64 & $-9.05 \times 10$ & $-1.30 \times 10^{-1}$ & $-1.19 \times 10^{-1}$ \\
\hline$b \bar{b}$ & $47 \times 10^{-1}$ & $4.29 \times 10^{-2}$ & -3.72 & $-4.15 \times 10^{-1}$ & -1.11 & 1.17 & 3.02 & $-0.95 \times 10^{-2}$ & $-1.30 \times 10^{-1}$ & $1.10 \times 10^{-1}$ \\
\hline$c \bar{c}$ & $2.25 \times 10^{-2}$ & $-1.07 \times 10^{-2}$ & -3.73 & $-4.38 \times 10^{-1}$ & -7.82 & -1.40 & 2.08 & 3.50 & $-1.30 \times 10^{-1}$ & $1.26 \times 10^{-1}$ \\
\hline$\tau^{+} \tau^{-}$ & $6.51 \times 10^{-2}$ & $8.22 \times 10^{-2}$ & -3.53 & $-4.68 \times 10^{-1}$ & 1.35 & -1.40 & 3.48 & $-0.95 \times 10^{-2}$ & 1.87 & $1.72 \times 10^{-1}$ \\
\hline$W W^{*}$ & $2.38 \times 10^{-1}$ & $-7.87 \times 10^{-2}$ & 9.14 & 1.38 & 1.36 & -1.40 & -8.63 & $-0.95 \times 10^{-2}$ & $-1.30 \times 10^{-1}$ & $-5.94 \times 10^{-1}$ \\
\hline$Z Z^{*}$ & $2.96 \times 10^{-2}$ & $5.76 \times 10^{-2}$ & $1.08 \times 10^{1}$ & $-8.11 \times 10^{-1}$ & 1.35 & -1.40 & -7.69 & $-0.95 \times 10^{-2}$ & $-1.30 \times 10^{-1}$ & 1.69 \\
\hline$Z \gamma$ & $1.74 \times 10^{-3}$ & $1.94 \times 10^{-2}$ & 6.53 & $-6.40 \times 10^{-2}$ & 1.35 & -1.41 & -1.32 & $-0.95 \times 10$ & $-1.30 \times 10^{-1}$ & 1.78 \\
\hline$\mu^{+} \mu^{-}$ & $2.25 \times 10^{-4}$ & $8.32 \times 10^{-2}$ & -3.53 & $-4.91 \times 10^{-1}$ & 1.35 & -1.40 & 3.48 & $-0.95 \times 10^{-2}$ & $-1.30 \times 10^{-1}$ & $1.73 \times 10^{-1}$ \\
\hline
\end{tabular}
For this reason, it is often useful [28] to measure the ratio of observables $\left(\sigma B_{1}\right) /\left(\sigma B_{2}\right) \simeq B_{1} / B_{2}$, where the

TABLE V. The reference value and expansion coefficients for Higgs boson decay branching fractions according to Eq. (12). The input parameters for this computation are from Table I. $V V^{*}$ partial decay widths are calculated by Prophecy $4 f$. 
TABLE VI. The percentage uncertainties of branching fractions due to uncertainties in each of the input observables, as calculated by Eq. (14). The input parameters for this computation are from Table I. In addition, we also compute the branching ratio uncertainties due to $\Delta m_{h}=0.1 \mathrm{GeV}$, the expected uncertainty after the LHC run. These values are in parentheses in the $\Delta_{m_{H}}$ column. Percentages less than $0.1 \%$ are listed as $\cdots$.

\begin{tabular}{|c|c|c|c|c|c|c|c|c|c|}
\hline & $\Delta_{m_{t}}$ & $\Delta_{m_{H}}$ & $\Delta_{\alpha\left(M_{Z}\right)}$ & $\Delta_{\alpha_{S}\left(M_{Z}\right)}$ & $\Delta_{m_{b}}$ & $\Delta_{M_{Z}}$ & $\Delta_{m_{c}}$ & $\Delta_{m_{\tau}}$ & $\Delta_{G_{I}}$ \\
\hline$g g$ & 0.07 & $0.52(0.13)$ & 0.01 & 2.27 & 1.84 & 0.01 & 0.38 & $\ldots$ & $\ldots$ \\
\hline$\gamma \gamma$ & $\ldots$ & $0.07(0.02)$ & 0.03 & 0.81 & 1.74 & $\ldots$ & 0.33 & $\ldots$ & $\ldots$ \\
\hline$b \bar{b}$ & 0.02 & $1.18(0.30)$ & 0.01 & 0.66 & 1.47 & 0.01 & 0.38 & $\ldots$ & $\ldots$ \\
\hline$c \bar{c}$ & 0.01 & $1.19(0.30)$ & 0.01 & 4.62 & 1.75 & $\ldots$ & 14.66 & $\ldots$ & $\ldots$ \\
\hline$\tau^{+} \tau^{-}$ & 0.05 & $1.12(0.28)$ & 0.01 & 0.80 & 1.75 & 0.01 & 0.38 & 0.02 & $\ldots$ \\
\hline$W W^{*}$ & 0.05 & $2.91(0.73)$ & 0.02 & 0.80 & 1.75 & 0.02 & 0.38 & $\ldots$ & $\ldots$ \\
\hline$Z Z^{*}$ & 0.03 & $3.43(0.86)$ & 0.01 & 0.80 & 1.75 & 0.02 & 0.38 & $\cdots$ & $\ldots$ \\
\hline$Z \gamma$ & 0.01 & $2.08(0.52)$ & $\ldots$ & 0.80 & 1.76 & $\ldots$ & 0.38 & $\ldots$ & $\ldots$ \\
\hline$\mu^{+} \mu^{-}$ & 0.05 & $1.12(0.28)$ & 0.01 & 0.80 & 1.75 & 0.01 & 0.38 & $\cdots$ & $\ldots$ \\
\hline
\end{tabular}

uncertainties in the production cross section largely drop out. It is beyond the purpose and scope of this paper to detail this process, but what we can do now is give accurate computations of the uncertainties of the ratios of branching fractions.

As with the partial widths and the branching fractions themselves, it is useful to expand the ratio of the branching fractions in the following form:

$$
\frac{\mathrm{B}(H \rightarrow \mathrm{X})}{\mathrm{B}(H \rightarrow \mathrm{Y})}=\frac{\mathrm{B}(\mathrm{X})^{(\mathrm{ref})}}{\mathrm{B}(\mathrm{Y})^{(\mathrm{ref})}}\left(1+\sum_{i} r_{\tau_{i}, X, Y} \delta \bar{\tau}_{i}\right)
$$

where $\tau_{i}$ represent the same parameters as Eq. (1). The expansion parameter $r_{\tau_{i}, X, Y}$ is related to $a_{\tau_{i}, X}$ by

$$
r_{\tau_{i}, X, Y}=a_{\tau_{i}, X}-a_{\tau_{i}, Y} .
$$

Using the reference parameters from Table I, we display the results of the reference ratio of the branching ratios and their deviations in Table VIII. Table IX presents the uncertainties of the predictions for these observables. We see that typically there is a few percent uncertainty in predicting the ratios of branching ratios, and, as emphasized above, these may be the cleanest observables the LHC experiment will present for some time.

\section{IMPLICATIONS FOR HIGGS BOSON STUDIES IN THE STANDARD MODEL AND BEYOND}

In this article we have done state-of-the art computations to detail the partial widths and branching fractions of the SM Higgs boson of $126 \mathrm{GeV}$. We have provided equations that Taylor expand the result about a set of input observables to show the shift in the partial width and branching fractions as a function of small deviations, including small deviations of the Higgs boson around $126 \mathrm{GeV}$.

The purpose of computing Higgs boson properties is to enable precision comparisons of data with theory. Up to the present time, the experimental uncertainties for Higgs boson physics are much larger than the uncertainties of the theoretical computations. Furthermore, the SM predicted rates for Higgs observables are well within the bands of experimental measurements.

Over time, however, the situation will change. It is hoped that experimental measurements will increase in precision so as to test any new physics contributions that might be influencing Higgs boson observables. The new physics contributions may be rather small and on the percent level [29], and so it behooves us to come to an understanding of how precisely one can really test the SM Higgs boson couplings. Our analysis can be used to address that question as well.

For example, if the data at a later stage of the LHC, ILC, or CLIC suggests that the branching fraction into $b$ quarks can be determined to better than $1 \%$, this does not mean that we are sensitive to new physics contributions of $1 \%$ to $H \rightarrow b \bar{b}$. It can be seen in Tables VI and VII that the SM uncertainty in computing $B(H \rightarrow b \bar{b})$ is presently $3.7 \%$

TABLE VII. The estimates for theory error (percent relative uncertainty) of the branching fractions due to parametric uncertainties and due to scale-dependent uncertainties from varying $m_{H} / 2 \leq \mu \leq 2 m_{H}$. Errors below $0.01 \%$ are reported in the table as 0 . For parametric uncertainties, we put an additional number in parentheses, which is the value it would have if the Higgs boson mass uncertainty were $0.1 \mathrm{GeV}$ (instead of $0.4 \mathrm{GeV})$.

\begin{tabular}{lccc}
\hline \hline & $P_{\mathrm{BR}}^{ \pm}$(par.-add. $)$ & $P_{\mathrm{BR}}^{ \pm}$(par.-quad. $)$ & $\left(P_{\mathrm{BR}}^{+}, P_{\mathrm{BR}}^{-}\right)(\mu)$ \\
\hline$g g$ & $5.10(4.71)$ & $2.99(2.95)$ & $(0.01,1.22)$ \\
$\gamma \gamma$ & $3.03(2.98)$ & $1.96(1.96)$ & $(1.80,1.81)$ \\
$b \bar{b}$ & $3.73(2.84)$ & $2.03(1.68)$ & $(0.24,0.00)$ \\
$c \bar{c}$ & $22.24(21.35)$ & $15.52(15.48)$ & $(0.52,0.38)$ \\
$\tau^{+} \tau^{-}$ & $4.13(3.29)$ & $2.26(1.98)$ & $(0.08,0.05)$ \\
$W W^{*}$ & $5.93(3.75)$ & $3.51(2.10)$ & $(0.09,0.06)$ \\
$Z Z^{*}$ & $6.42(3.85)$ & $3.95(2.14)$ & $(0.09,0.06)$ \\
$Z \gamma$ & $5.04(3.48)$ & $2.87(2.04)$ & $(0.83,0.78)$ \\
$\mu^{+} \mu^{-}$ & $4.12(3.27)$ & $2.26(1.98)$ & $(0.07,0.04)$ \\
\hline \hline
\end{tabular}



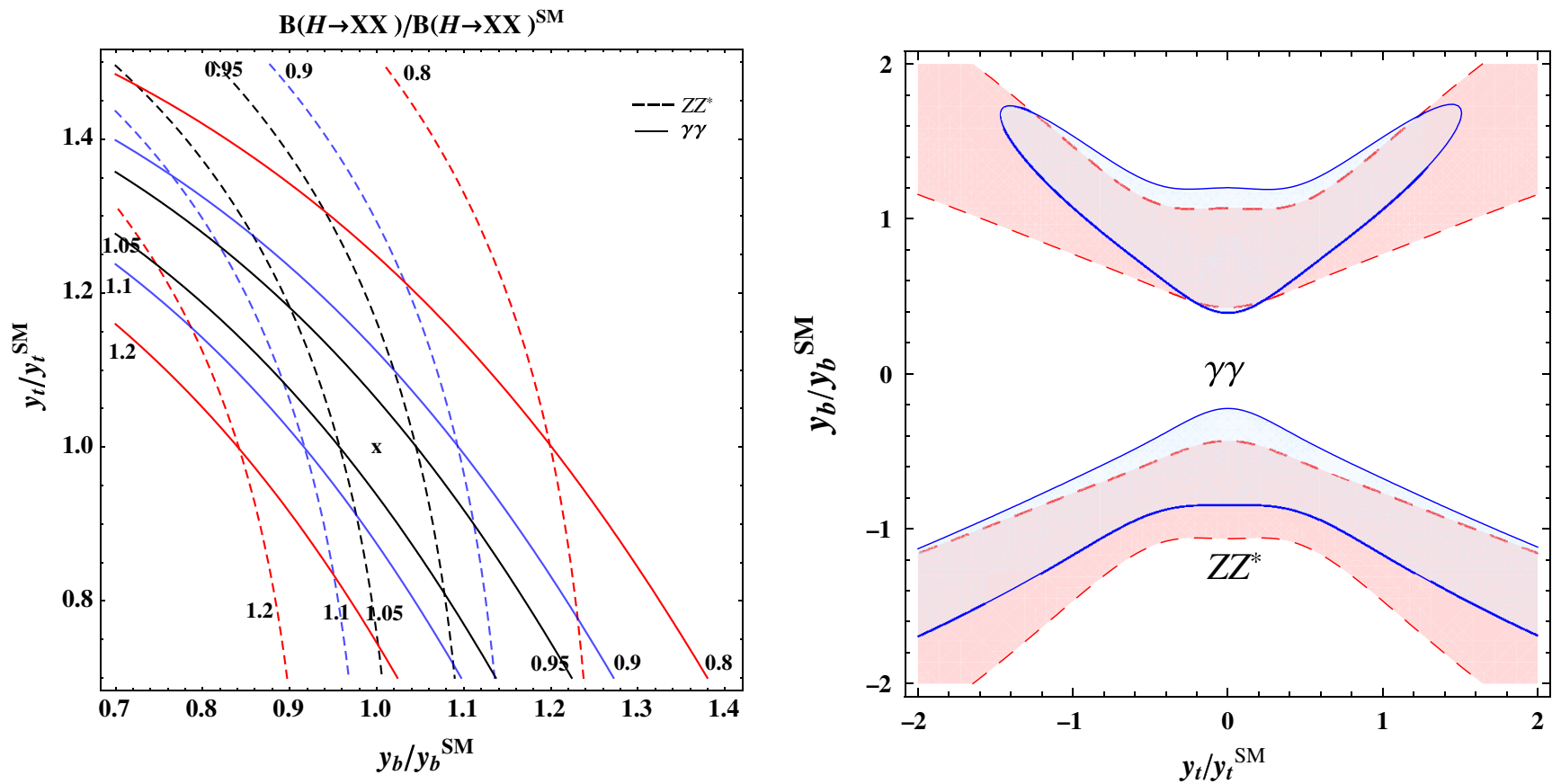

FIG. 1 (color online). Left Panel: Contours of $B(H \rightarrow \gamma \gamma) / B(H \rightarrow \gamma \gamma)_{\mathrm{SM}}$ (solid lines) and $B(H \rightarrow Z Z) / B(H \rightarrow Z Z)_{\mathrm{SM}}($ dashed lines) in the $y_{t}-y_{b}$ plane. The SM position at $(1,1)$ is marked with an $\mathrm{x}$. Right Panel: The red shaded region is the $1 \sigma$ allowed region for $y_{t} / y_{t}^{s m}$ and $y_{b} / y_{b}^{s m}$ given current data limits on $\sigma(H) \times B\left(H \rightarrow Z Z^{*}\right)$. The blue shaded region is the current $1 \sigma$ allowed region from current data limits on $\sigma(H) \times B(H \rightarrow \gamma \gamma)$.

(sum of absolute values of all errors), and it is expected not to get better than $2.8 \%$, with most of that coming from uncertainty of the bottom Yukawa coupling determination stemming from the uncertainty of the measured bottom quark pole mass, and the theory uncertainties encountered when extracting and connecting the two. Thus, without reducing this error, any new physics contribution to the $b \bar{b}$ branching fraction that is not at least a factor of 2 or 3 larger than $2 \%$ cannot be discerned. Thus, a deviation of at least $5 \%$ is required of detectable new physics.

Some new physics ideas shift the $b$-quark Yukawa coupling away from the assumed SM value by virtue of the added contributions from induced finite $b$-quark mass corrections. An example of this is in high $\tan \beta$ supersymmetric theories. A relative shift in the $b$ quark Yukawa coupling $y_{b} \rightarrow y_{b}\left(1+\delta_{b}\right)$ translates into a shift of the branching fraction by $\delta B_{b}=2 B_{b}\left(1-B_{b}\right) \delta_{b} \simeq \delta_{b} / 2$. Thus, one would have to shift the bottom Yukawa coupling by more than $10 \%$ to have any hope of discerning a non-SM signal.

In the left panel of Fig. 1, we show contours of $B(H \rightarrow$ $\gamma \gamma) / B(H \rightarrow \gamma \gamma)_{\mathrm{SM}}$ (solid lines) and $B(H \rightarrow Z Z) / B(H \rightarrow$ $Z Z)_{\mathrm{SM}}$ (dashed lines) in the $y_{t}-y_{b}$ plane, assuming that new physics only shifts the Yukawa couplings of the third generation fermions $t$ and $b$. The SM value for each is determined at the $\mathrm{x}$ position in the center of the figures where $y_{f} / y_{f}^{\mathrm{SM}}=1$. At this point, the values are $B(H \rightarrow$ $\left.Z Z^{*}\right)=0.030$ and $B(H \rightarrow \gamma \gamma)=0.0027$. The $1 \sigma$ relative uncertainty of the SM calculation for $\Delta B\left(Z Z^{*}\right)$ is about
$4.0 \%$ and for $\Delta B(\gamma \gamma)$ about $2.0 \%$ (see Table VII). These uncertainties cut large yet finite-width constraining areas in the plane of the left panel of Fig. 1. Furthermore, for correlated values of $\delta y_{b}$ and $\delta y_{t}$ shifts, there is no shift at all in these branching ratios. Nevertheless, the contours of constant $B(H \rightarrow \gamma \gamma)$ have different slope than the contours of $B(H \rightarrow Z Z)$, enabling determinations of $y_{t}$ and $y_{b}$ from a combination of precise measurements of these two observables alone.

In the right panel of Fig. 1, we demonstrate limits on $y_{t}$ and $y_{b}$ more directly from LHC data. The red shaded region is the $1 \sigma$ allowed region for $y_{t} / y_{t}^{s m}$ and $y_{b} / y_{b}^{s m}$ given current data limits on $\sigma(H) \times B\left(H \rightarrow Z Z^{*}\right)$. The blue shaded region is the current $1 \sigma$ allowed region from current data limits on $\sigma(H) \times B(H \rightarrow \gamma \gamma)$. The overlap region of these two shaded regions is the first estimate of where a global fit to the data suggests $y_{t}$ and $y_{b}$ must be. The $\gamma \gamma$ and $\mathrm{ZZ}$ observables are the most powerful ones at present, and so it is appropriate to use them as illustration.

As we can see, the data allow increasing $y_{b}$ as long as $y_{t}$ is increasing. This can be understood as the cancellation of two effects. When $y_{b}$ increases, the branching ratio to $b b$ increases, and therefore the branching fraction to $\gamma \gamma$ and $Z Z^{*}$ diminishes. However, if $y_{t}$ increases, then the production cross section $\sigma(g g \rightarrow H)$ increases, due to its primary contribution from a top quark loop diagram. It is these considerations that yield the shape of the allowed shaded regions in the right panel of Fig. 1. In the case of $\gamma \gamma$, an ever increasing positive $y_{b}$ and positive $y_{t}$ is not without 
TABLE VIII. The reference values and expansion coefficients for ratios of Higgs boson decay branching fractions according to Eq. (16). The input parameters for this computation are from Table I. $V V^{*}$ partial decay widths are calculated by Prophecy $4 f$.

\begin{tabular}{|c|c|c|c|c|c|c|c|c|c|c|}
\hline & $B(X) / B(Y)_{\operatorname{Ref}}$ & $r_{m_{t}}$ & $r_{m_{H}}$ & $r_{\alpha\left(M_{Z}\right)}$ & $r_{\alpha_{S}\left(M_{Z}\right)}$ & $r_{m_{b}}$ & $r_{M_{Z}}$ & $r_{m_{c}}$ & $r_{m_{\tau}}$ & $r_{G_{F}}$ \\
\hline$\overline{\gamma \gamma / W W^{*}}$ & $1.15 \times 10^{-2}$ & $8.62 \times 10^{-2}$ & -9.35 & $4.60 \times 10^{-1}$ & $4.60 \times 10^{-1}$ & $9.01 \times 10^{-3}$ & $1.03 \times 10^{1}$ & 0 & 0 & $4.75 \times 10^{-1}$ \\
\hline$b \bar{b} / c \bar{c}$ & $2.17 \times 10^{1}$ & $5.36 \times 10^{-2}$ & $9.6 \times 10^{-3}$ & $2.24 \times 10^{-2}$ & 6.71 & 2.57 & $9.34 \times 10^{-1}$ & -3.59 & 0 & $1.66 \times 10^{-2}$ \\
\hline$\tau^{+} \tau^{-} / \mu^{+} \mu^{-}$ & $2.89 \times 10^{2}$ & $-1.03 \times 10^{-3}$ & $2.55 \times 10^{-3}$ & $2.22 \times 10^{-2}$ & $4.65 \times 10^{-5}$ & 0 & $1.09 \times 10^{-4}$ & 0 & 2.01 & $-3.39 \times 10^{-4}$ \\
\hline$c \bar{c} / \mu^{+} \mu^{-}$ & $1.12 \times 10^{2}$ & $-9.39 \times 10^{-2}$ & $-1.93 \times 10^{-1}$ & $5.29 \times 10^{-2}$ & -9.17 & 0 & -1.39 & 3.59 & 0 & $-4.64 \times 10^{-2}$ \\
\hline$W W^{*} / Z Z^{*}$ & 8.05 & $-1.36 \times 10^{-1}$ & -1.63 & 2.19 & $1.09 \times 10^{-2}$ & 0 & $-9.38 \times 10^{-1}$ & 0 & 0 & -2.28 \\
\hline$\gamma \gamma / Z Z^{*}$ & $9.22 \times 10^{-2}$ & $-5.02 \times 10^{-2}$ & $-1.1 \times 10^{1}$ & 2.65 & $1.98 \times 10^{-2}$ & $9.01 \times 10^{-3}$ & 9.33 & 0 & 0 & -1.81 \\
\hline$b \bar{b} / Z Z^{*}$ & $1.85 \times 10^{1}$ & $-1.47 \times 10^{-2}$ & $-1.45 \times 10^{1}$ & $3.96 \times 10^{-1}$ & -2.46 & 2.57 & $1.07 \times 10^{1}$ & 0 & 0 & -1.58 \\
\hline$\tau^{+} \tau^{-} / Z Z^{*}$ & 2.2 & $2.46 \times 10^{-2}$ & $-1.43 \times 10^{1}$ & $3.43 \times 10^{-1}$ & $-3.29 \times 10^{-4}$ & 0 & $1.12 \times 10^{1}$ & 0 & 2.01 & -1.52 \\
\hline$Z \gamma / Z Z^{*}$ & $5.87 \times 10^{-2}$ & $-3.82 \times 10^{-2}$ & -4.23 & $7.47 \times 10^{-1}$ & $1.82 \times 10^{-3}$ & $-9.76 \times 10^{-3}$ & 6.37 & 0 & 0 & $8.96 \times 10^{-2}$ \\
\hline$b \bar{b} / \tau^{+} \tau^{-}$ & 8.41 & $-3.93 \times 10^{-2}$ & $-1.86 \times 10^{-1}$ & $5.31 \times 10^{-2}$ & -2.46 & 2.57 & $-4.59 \times 10^{-1}$ & 0 & 0 & $-6.26 \times 10^{-2}$ \\
\hline$\tau^{+} \tau^{-} / c c$ & 2.58 & $9.29 \times 10^{-2}$ & $1.96 \times 10^{-1}$ & $-3.07 \times 10^{-2}$ & 9.17 & 0 & 1.39 & -3.59 & 2.01 & $4.61 \times 10^{-2}$ \\
\hline$\gamma \gamma / Z \gamma$ & 1.57 & $-1.19 \times 10^{-2}$ & -6.74 & 1.90 & $1.80 \times 10^{-2}$ & $1.88 \times 10^{-2}$ & 2.96 & 0 & 0 & -1.90 \\
\hline$g g / Z_{\gamma}$ & $3.31 \times 10^{1}$ & $-1.47 \times 10^{-1}$ & -8.17 & $-3.81 \times 10^{-1}$ & 2.49 & $-6.12 \times 10^{-2}$ & 5.19 & 0 & 0 & -1.62 \\
\hline
\end{tabular}

bound because the production cross section and branching fraction both increase. However, for positive $y_{b}$ and negative $y_{t}$, and vice versa, there can be no bound since the production cross-section gains can be countered by the branching fraction losses, due to destructive interference, and vice versa. In all cases, there is still room for quite sizable shifts in the top and bottom quark couplings to the Higgs boson while remaining consistent with the data.

In conclusion, inspection of Tables VII and IX suggests that among most branching ratios, and among most ratios of branching ratios, the SM value cannot be determined theoretically to within better than a few percent. When considering a future precision Higgs boson program at the LHC or another collider experiment beyond it, with hopes of getting measurements at the percent level or better to test new physics ideas, it will become necessary to confront the theory and input observable uncertainties that plague

TABLE IX. The estimates for theory error (percent relative uncertainty) of the ratio of branching fractions due to parametric uncertainties and due to scale-dependent uncertainties from varying $m_{H} / 2 \leq \mu \leq 2 m_{H}$. Errors below $0.01 \%$ are reported in the table as 0 . For parametric uncertainties, we put an additional number in parentheses, which is the value it would have if the Higgs boson mass uncertainty were $0.1 \mathrm{GeV}$ (instead of $0.4 \mathrm{GeV})$.

\begin{tabular}{lccc}
\hline \hline & $P^{ \pm}$(par.-add. $)$ & $P^{ \pm}$(par.-quad. $)$ & $\left(P^{+}, P^{-}\right)(\mu)$ \\
\hline$\gamma \gamma / W W^{*}$ & $3.34(1.11)$ & $2.99(0.79)$ & $(1.71,1.75)$ \\
$b \bar{b} / c \bar{c}$ & $22.27(22.26)$ & $15.89(15.89)$ & $(0.62,0.41)$ \\
$\tau^{+} \tau^{-} / \mu^{+} \mu^{-}$ & $0.02(0.02)$ & $0.02(0.02)$ & $(0.02,0.02)$ \\
$c \bar{c} / \mu^{+} \mu^{-}$ & $20.58(20.54)$ & $15.99(15.99)$ & $(0.46,0.34)$ \\
$W W^{*} / Z Z^{*}$ & $0.64(0.25)$ & $0.52(0.16)$ & $(0 ., 0)$. \\
$\gamma \gamma / Z Z^{*}$ & $3.61(0.99)$ & $3.49(0.88)$ & $(1.71,1.75)$ \\
$b \bar{b} / Z Z^{*}$ & $9.32(5.87)$ & $5.81(3.72)$ & $(0.31,0.02)$ \\
$\tau^{+} \tau^{-} / Z Z^{*}$ & $4.61(1.20)$ & $4.55(1.14)$ & $(0.01,0.01)$ \\
$Z \gamma / Z Z^{*}$ & $1.41(0.40)$ & $1.35(0.34)$ & $(0.73,0.71)$ \\
$b \bar{b} / \tau^{+} \tau^{-}$ & $4.76(4.71)$ & $3.53(3.53)$ & $(0.30,0.01)$ \\
$\tau^{+} \tau^{-} / c \bar{c}$ & $20.60(20.55)$ & $15.99(15.99)$ & $(0.33,0.44)$ \\
$\gamma \gamma / Z \gamma$ & $2.22(0.61)$ & $2.15(0.54)$ & $(0.97,1.04)$ \\
$g g / Z \gamma$ & $4.25(2.30)$ & $2.99(1.61)$ & $(0.79,2.94)$ \\
\hline \hline
\end{tabular}

further improvement. We believe that the expansion technique presented in this paper is the most up-to-date presentation of partial width and branching ratio observable calculations in the SM, that it is ideal for investigating consequences of physics beyond the SM, and that it most clearly shows the precise areas of improvement needed for SM calculations.

\section{ACKNOWLEDGMENTS}

We thank K. Chetyrkin for helpful communications. We thank A. Mueck, S. Heinemeyer, I. Puljak, and D. Rebuzzi for drawing our attention to corrections to our original $\alpha_{s}$ dependencies. S. L. is supported by the National Research Foundation of Korea Grant No. 1120547 funded by the Korea government. L. A. is supported by the P2IO Labex. S. P. is supported by National Science Centre under Research Grants No. DEC-2011/01/M/ST2/02466, No. DEC-2012/04/A/ST2/00099, and No. DEC-2012/05/ B/ST2/02597. L. A. and S. L. would also like to thank the CERN Theory Group for their hospitality, where part of this work was done. This work was supported by the Supercomputing Center/Korea Institute of Science and Technology Information with supercomputing resources including technical support pd0326.

\section{APPENDIX A}

In this appendix, we give the scale dependence to scalar correlator, $R_{S}\left(\mu^{2}, m_{h}^{2}\right)$ at $\mathcal{O}\left(\alpha_{S}^{4}\right)$. This contributes to the decay of the Higgs boson into heavy quarks in the following form:

$$
\Gamma_{H \rightarrow Q \bar{Q}}\left(\mu^{2}\right)=\bar{\sigma}_{0} m_{Q}^{2}\left(\mu^{2}\right) R_{S}\left(\frac{\mu^{2}}{m_{h}^{2}}\right)
$$

where $\overline{\sigma_{0}}$ is the lowest-order cross section without the outgoing quark masses. The scale invariance of $\Gamma_{H \rightarrow Q \bar{Q}}\left(\mu^{2}\right)$, together with the results for $R_{S}$ at $\mu=m_{h}$ to $\mathcal{O}\left(\alpha_{S}^{4}\right)$ given in [21], leads to the following additional $\alpha_{S}^{4}$ contributions to $R_{S}\left(\mu^{2}, m_{h}^{2}\right)$ previously not found in the literature. 


$$
\begin{aligned}
R_{S}\left(\mu^{2}, m_{h}^{2}\right)= & 3\left\{1+\left(\frac{\alpha_{S}(\mu)}{\pi}\right)\left(5.666+2 \log \left(\frac{\mu^{2}}{m_{h}^{2}}\right)\right)+\left(\frac{\alpha_{S}(\mu)}{\pi}\right)^{2}\left(29.1467+29.222 \log \left(\frac{\mu^{2}}{m_{h}^{2}}\right)+3.917 \log ^{2}\left(\frac{\mu^{2}}{m_{h}^{2}}\right)\right)\right. \\
& +\left(\frac{\alpha_{S}(\mu)}{\pi}\right)^{3}\left(41.758+185.295 \log \left(\frac{\mu^{2}}{m_{h}^{2}}\right)+90.545 \log ^{2}\left(\frac{\mu^{2}}{m_{h}^{2}}\right)+7.616 \log ^{3}\left(\frac{\mu^{2}}{m_{h}^{2}}\right)^{3}\right) \\
& \left.+\left(\frac{\alpha_{S}(\mu)}{\pi}\right)^{4}\left(-825.7+443.937 \log \left(\frac{\mu^{2}}{m_{h}^{2}}\right)+721.581 \log ^{2}\left(\frac{\mu^{2}}{m_{h}^{2}}\right)+238.608 \log ^{3}\left(\frac{\mu^{2}}{m_{h}^{2}}\right)+14.755 \log ^{4}\left(\frac{\mu^{2}}{m_{h}^{2}}\right)\right)\right\}
\end{aligned}
$$

The coefficients are given at $N_{c}=3, n_{f}=5$ since these are where the Standard Model contributes and where the values at $\mu=m_{h}$ are reported. These contribute to an $\mathcal{O}(0.01 \%)$ scale uncertainty to the width results.

[1] G. Aad et al. (ATLAS Collaboration), Phys. Lett. B 716, 1 (2012); S. Chatrchyan et al. (CMS Collaboration), Phys. Lett. B 716, 30 (2012).

[2] A. Djouadi, J. Kalinowski, and M. Spira, Comput. Phys. Commun. 108, 56 (1998).

[3] For an earlier study with some overlap in goals, see A. Denner, S. Heinemeyer, I. Puljak, D. Rebuzzi, and M. Spira, Eur. Phys. J. C 71, 1753 (2011).

[4] A. Djouadi, Phys. Rep. 457, 1 (2008).

[5] See, for example, Sec. 8.2 of S. Schael et al. (LEP Electroweak Working Group), Phys. Rep. 427, 257 (2006).

[6] CMS Collaboration, Report No. CMS PAS HIG-13-005.

[7] J. Beringer et al. (Particle Data Group), Phys. Rev. D 86, 010001 (2012).

[8] A. Ferroglia, G. Ossola, M. Passera, and A. Sirlin, Phys. Rev. D 65, 113002 (2002).

[9] As an example, see the recent study of J. R. Espinosa, C. Grojean, M. Muhlleitner, and M. Trott, J. High Energy Phys. 05, (2012) 097.

[10] W.-Y. Keung and W. J. Marciano, Phys. Rev. D 30, 248 (1984).

[11] A. Bredenstein, A. Denner, S. Dittmaier, and M. M. Weber, Phys. Rev. D 74, 013004 (2006).

[12] A. Bredenstein, A. Denner, S. Dittmaier, and M. M. Weber, arXiv:0708.4123.

[13] P. Maierhofer and P. Marquard, arXiv:1212.6233.
[14] S. Actis, G. Passarino, C. Sturm, and S. Uccirati, Phys. Lett. B 670, 12 (2008).

[15] K. G. Chetyrkin, J. H. Kuhn, and M. Steinhauser, Comput. Phys. Commun. 133, 43 (2000).

[16] M. Spira, A. Djouadi, and P. M. Zerwas, Phys. Lett. B 276, 350 (1992).

[17] M. Schreck and M. Steinhauser, Phys. Lett. B 655, 148 (2007).

[18] K. G. Chetyrkin, B. A. Kniehl, and M. Steinhauser, Phys. Rev. Lett. 79, 2184 (1997).

[19] M. Steinhauser, Phys. Rep. 364, 247 (2002).

[20] S. Actis, G. Passarino, C. Sturm, and S. Uccirati, Nucl. Phys. B811, 182 (2009).

[21] P. A. Baikov, K. G. Chetyrkin, and J. H. Kuhn, Phys. Rev. Lett. 96, 012003 (2006).

[22] M. Butenschoen, F. Fugel, and B. A. Kniehl, Nucl. Phys. B772, 25 (2007).

[23] K. G. Chetyrkin, Phys. Lett. B 390, 309 (1997).

[24] K. G. Chetyrkin and M. Steinhauser, Phys. Lett. B 408, 320 (1997).

[25] A. Dabelstein and W. Hollik, Z. Phys. C 53, 507 (1992).

[26] B. A. Kniehl, Nucl. Phys. B376, 3 (1992).

[27] B. A. Kniehl and M. Steinhauser, Phys. Lett. B 365, 297 (1996).

[28] See, for example, A. Djouadi, and G. Moreau, arXiv:1303.6591.

[29] See, for example, R. S. Gupta, H. Rzehak, and J. D. Wells, Phys. Rev. D 86, 095001 (2012). 